\title{
Homomorphisms of Knot Groups on Finite Groups
}

\author{
By Robert Riley
}

\begin{abstract}
We describe trial and error computer programs for finding certain homomorphisms of a knot group on a special projective group $\operatorname{LF}(2, p), p$ prime, and programs to evaluate $H_{1}(\Re) ; Z$ ) where $\Re\left(\right.$ is a finitely sheeted branched covering space of $S^{3}$ associated with such a homomorphism. These programs have been applied to several collections of examples, in particular to the Kinoshita-Terasaka knots, and we state numerous conjectures based on these experiments.
\end{abstract}

About forty years ago a universal method for obtaining algebraic invariants of knot type was proposed and became standard. The method, as applied to a knot $k$ of type $K$ and having group $\pi K=\pi_{1}\left(S^{3}-k ; *\right)$, begins with the determination of the homomorphisms of $\pi K$ on a given group $G$. These homomorphisms fall into equivalence classes under the action of the automorphisms of $G$, and a crude preliminary invariant of $K$ is the number of homomorphism classes. In the next stage of the method, we fix a transitive permutation representation of $G$, perhaps of infinite degree. Each homomorphism class of $\pi K$ on $G$ is associated with a covering space $\mathcal{u}$ of $S^{3}-k$ such that the number of sheets in the covering is the degree of the permutation representation, and the group $H_{1}(\mathcal{U} ; Z)$ is an algebraic invariant of the knot type $K$. In this paper, we shall discuss the means and results of implementing the universal method on a computer when the group $G$ is chosen to be one of the special projective groups $L_{p}=L F(2, p)=\operatorname{PSL}(2, p)$, where $p$ is a prime integer.

The universal method has been most thoroughly examined in the case where the group $G$ is cyclic. The determination of the homomorphism classes becomes completely trivial, and all the homology invariants can be deduced from a single matrix, the Alexander matrix. These "cyclic invariants" have been applied with good effect to just about every problem in knot theory, but, alas, when the Alexander polynomial $\Delta(x)$ of the knot reduces to the constant 1 these invariants degenerate and are worthless. It is no good choosing a solvable group for $G$ in such a case; to get useful results from the universal method, we must use nonsolvable groups. Of course, simple groups receive first consideration in this context, and of the families of classical finite simple groups, the family $\left\{L_{p}\right\}$ is the most manageable. As further encouragement for this study, the group $L_{5}$ is isomorphic to the alternating group $A_{5}$, and R. H. Fox has shown in several papers ([5] is a good example) that the homomorphisms of a knot group on $A_{5}$ have some interesting applications. There are, however, very few clues to suggest what the best method for finding homomorphisms on the special projective groups could be. In addition, almost nothing is known about the homology invariants of homomorphisms on noncyclic groups $G$. It therefore seems reasonable

Received July 17, 1970, revised December 2, 1970.

AMS 1969 subject classifications. Primary 5520.

Key words and phrases. Homomorphisms of knot groups on finite groups, homology of covering spaces.

Copyright (C) 1971, American Mathematical Society 
to explore the subject initially by applying the most humble methods to a large number of examples. This leads directly to the development of computer programs to do the work and to large tables of output data. These are the subject of this paper.

Our method for finding the homomorphisms of a knot group $\pi K$ on a finite group $G$ is simple trial and error. Suppose that $\pi K$ is given to us in the form

$$
\pi K=\left|x_{1}, \cdots, x_{n}: r_{1}(\mathbf{x})=r_{2}(\mathbf{x})=\cdots=1\right| \text {. }
$$

Let $\mathrm{a}=\left(a_{1}, \cdots, a_{n}\right)$ be a set of elements of $G$ which together generate $G$. The assignment $x_{i} \rightarrow a_{i}$, for $i=1, \cdots, n$, can be extended to a homomorphism $\theta: \pi K \rightarrow G$ such that $\left(x_{i}\right) \theta=a_{i}$ if and only if $r_{1}(\mathrm{a})=r_{2}(\mathrm{a})=\cdots=1$. Hence the determination of the homomorphisms (and later their arrangement into classes) is a finite process. But before we actually carry out this process even on a computer, we should find ways of reducing the number of experiments to the irreducible minimum. When the presentation (0) is an over-presentation (see [5] or [7]), the generators $x_{i}$ are all conjugate in $\pi K$. This is such a great aid to the classification that we use only overpresentations for knot groups. In Section 4, we discuss a version of the overpresentation that is especially well adopted to our methods and programs.

In Section 2, we determine the irreducible set of experiments needed to determine the homomorphism classes of $\pi K$ on $A_{5}$ when $n$ in (0) is 2 or 3. It turns out that there are three possible kinds of such homomorphisms, and that the search for one kind is much easier to describe and implement in a computer program than the others. The characteristic property of these homomorphisms is that the image of an overgenerator of $(0)$ is an element of order 5 in $A_{5}$. In addition, we found that such homomorphisms are more numerous than the others for our test cases. Hence, when we generalize the $A_{5}$ case to $L_{p}$ in Section 3, we restrict ourselves to the homomorphisms which carry an over-generator of $\pi K$ to an element of order $p$ in $L_{p}$. We call such homomorphisms "reps" for brevity, and note that the property of being a rep is invariant, that is, it does not depend on the over-presentation (0) used in the definition. In Section 5, we discuss how to implement the second stage of the universal method on a computer when $G$ is an arbitrary finite group. Our tools are now ready.

In Section 6, we discuss the outcome of applying our programs to various collections of test cases. We have concentrated most of our effort on the groups $G=L_{p}$ where $p \leqq 11$, with the greatest emphasis on $p=5$ because the results are all new and the computation time per knot is still reasonable. The first set of test knots that we discuss are the prime knots with at most 9 crossing points, conveniently drawn on pp. 70-72 of Reidemeister [13]. We call these the classical knots and we have two tables in the microfiche section for them. The first table is of over-presentations for their groups which may be used as a standard reference by anyone doing computation in knot theory. The second table is of the invariants of these knots associated with their homomorphisms on $A_{5}=L_{5}$. We state a large number of conjectures about the homology invariants associated with reps of an arbitrary knot group on $L_{p}$ that are backed up by numerous tables of data which are not all discussed here. We hope that the reader shares the author's faith that only a small amount of wit and discrimination is needed to extract good conjectures from modest amounts of numerical data in this subject. The homology invariants for a certain special sort of reps are also discussed in Conjecture B, and we believe that this may be the best clue to the 
whole subject. The section ends with a discussion of three particular knots having Alexander polynomial $\Delta(x) \equiv 1$, namely Seifert's knot, the 11-crossing KinoshitaTerasaka knot [10], and a third 11-crossing knot discovered by J. Conway [2]. These three knots are shown to have different types.

In Section 7, we demonstrate the power and limitations of our programs by trying them on an infinite collection of knots that can be described by two integral parameters. These knots were discovered by Kinoshita and Terasaka [10] and shown to be nontrivial with $\Delta(x) \equiv 1$. We shall show that a homomorphism of the group of one of these knots on $A_{5}$ determines a corresponding homomorphism for the groups of an infinity of other knots whose integral parameters vary in certain arithmetic progressions. If we vary one of these integral parameters in its progression, we get interesting formulas for the corresponding homology invariants. If these formulas were proven (but it is not worth the effort to do this), they would show that these knots determine infinitely many types. As it is, we have only proven that they determine at least 200 types.

The work described in this paper has led to a later paper, "Parabolic representations of knot groups" that we are submitting for publication elsewhere. In it, we prove that many of the knot groups discussed here have representations on subgroups of $L F(2, \mathrm{C})$, from each of which one can derive an infinity of reps on the groups $L_{p}$.

I am indebted to Dr. G. Edmunds for his useful comments and constructive criticism, and to Professor W. Magnus for his kind encouragement of this project at a time when its continuation was in doubt.

1. Throughout this paper we shall use a standard notation for groups and homomorphisms taken from Huppert [9]. In particular, we write operators on the right so that products of permutations are read from left to right, when $x, y$ are elements of a group $x^{y} \equiv y^{-1} x y$, and $G=\left\langle x_{1}, \cdots, x_{n}\right\rangle$ means that $G$ is the group generated by $x_{1}, \cdots, x_{n}$.

All knots and knot types discussed in this paper are tame. We shall use the notation $k$ for a knot in $S^{3}, \bar{K}$ for its isotopy type, $\bar{K}^{\prime}$ for the isotopy type of a mirror image of $k$ and $K$ for the type of $k$, i.e. two knots $k_{1}, k_{2}$ in $S^{3}$ have the same type if there exists an autohomeomorphism of $S^{3}$ such that $\alpha\left(k_{1}\right)=k_{2}$. This notation is obviously consistent with subscripts and we use it with the understanding that mentioning any of $k_{i}, \bar{K}_{i}, \bar{K}_{i}^{\prime}$ or $K_{i}$ makes clear what the other symbols mean. The group of a knot, $\pi_{1}\left(S^{3}-k ; *\right)$, depends only on $K$ and we denote it $\pi K$. If $T$ is the boundary of a nice tubular neighbourhood of $k$ and $*_{T}$ is a point on $T$ the group $\pi_{1}\left(T ; *_{T}\right)$ determines a conjugacy class of subgroups of $\pi K$, and $\pi K$ with this conjugacy class specified will be called the marked group of $\bar{K}$. The simplest way to mark $\pi K$ is to name a longitude commuting with a chosen over-generator of $\pi K$, in practice always with the first named over generator.

Consider an over-presentation

$$
\pi K=\left|x_{1}, \cdots, x_{n}: r_{1}, \cdots, r_{n-1}\right|
$$

of the knot group $\pi K$. Each relation $r_{i}=1$ can be written as $x_{0}=W^{-1} x_{t} W$ where $W \in \pi K$. We can use such a relation to eliminate the generator $x_{0}$ whenever $W$ can be written without using $x_{2}$. By this means the original presentation (1) can be changed to a new presentation of the same form as (1), but with a smaller number of 
generators $n$. Of course, the remaining generators are still over-generators of $\pi K$ so they are still conjugate. We shall call such a presentation a normal presentation and we shall use only normal presentations in this paper. In Section 4 we will describe a modified version of a normal presentation which is more convenient in practice.

Among all the normal presentations of $\pi K$, there must be some written on the least number of generators. If this least number is $\nu$, then $\nu$ depends only on $K$ and we denote it by $\nu K=\nu$. It is not hard to see that $\nu K$ is the minimal bridge number [14] of $K$, but we make no use of its known properties. We do not know how to calculate $\nu K$; however, it is usually possible to find its true value by inspection even when there is no proof available. $\nu K$ must not be confused with the minimal number of generators for $\pi K$. For example, when $K$ is a torus knot, this minimal number of generators is 2, but $\nu K$ can be arbitrarily large [14, Satz 10].

The only knot $K$ with $\nu K=1$ is the trivial knot $K=0$. The class of knots $K$ with $\nu K=2$ has been completely classified in [15] and differently in [2]. These knots are also known as rational knots (Viergeflechte) and they are all of alternating type. There is probably no reasonable scheme to classify the knots with $\nu K=3$. A wild knot $K$ would have $\nu K=\infty$.

Let $\pi K$ be a knot group, $G$ a group, and consider the various homomorphisms $\theta_{1}, \theta_{2}, \cdots$ of $\pi K$ on $G$. We shall say that $\theta_{1}$ is equivalent to $\theta_{2}$, in symbols $\theta_{1} \equiv \theta_{2}$, when there exists an automorphism $\omega$ of $G$ such that $\theta_{2}=\theta_{1} \omega$. We shall also say that $\theta_{1}$ is weakly equivalent to $\theta_{2}$ if there is an automorphism $\alpha$ of $\pi K$ such that $\theta_{2} \equiv \alpha \theta_{1}$. Because the determination of the automorphism group of a knot group is an unsolved and presumably very knotty problem, we are forced to use equivalence much more than weak equivalence.

Let $G$ be a group of permutations of the numbers $1, \cdots, n$. We will write the elements $g$ of $G$ as permutations in two different ways. In the first way, we write $\left[a_{1}, \cdots, a_{n}\right]$ for the element $g \in G$ such that $i \cdot g=a_{i}$, for $i=1, \cdots, n$. In the second way, we write $g$ as a product of disjoint cycles.

$$
g=\left(b_{11}, b_{12}, \cdots, b_{1 r_{2}}\right)\left(b_{21}, b_{22}, \cdots, b_{2 r_{2}}\right) \cdots\left(b_{s 1}, b_{s 2}, \cdots, b_{r_{0}}\right)
$$

where $r_{1}+r_{2}+\cdots+r_{s}=n, b_{i j} g=b_{i j+1}\left(\operatorname{second}\right.$ index $\left.\left(\bmod r_{i}\right)\right)$, and each of $1, \cdots, n$ occurs in exactly one cycle. We shall omit cycles of length 1 from (2), and when $s=1$ we shall also omit the parentheses. In fact, we shall omit the commas from both representations wherever possible, e.g. [23451] $=12345$. We shall write $E$ for the identity $[123 \cdots n]$.

2. Let $\pi K$ be a knot group presented by a normal presentation (1) on $n=2$ or 3 generators. In this section, we shall reduce the problem of classifying the equivalence classes of homorphisms of $\pi K$ on $A_{5}$ to a reasonable number of experiments, viz. 3 when $n=2$, and 93 when $n=3$. We begin with some assertions about $A_{5}$ which are best proven by direct calculation.

The group $A_{5}$ has 60 elements, viz. 1 of order 1, 15 of order 2, 20 of order 3 and 24 of order 5 . All the elements of a given order except 5 are conjugate and the elements of order 5 lie in two conjugacy classes. If $C \in A_{5}$ has order 5 , then $\alpha$ is conjugate to $C, C^{-1}$ but not to $C^{2}, C^{-2}$. The automorphism group of $A_{5}$ is the symmetric group $S_{5}$ acting by conjugation, viz. let $a \in S_{5}, g \in A_{5}$, then $g \alpha=g^{\alpha}$. All the elements of $A_{5}$ of a given order are equivalent under the action of $S_{5}$. The maximal 
subgroups of $A_{5}$ are the stabilizers of a symbol 1, $\cdots, 5$ (these subgroups are isomorphic to $A_{4}$ ), and dihedral groups of order 10 . If two elements of $A_{5}$ commute, they either lie in a cyclic subgroup or they have order 2 and lie in the stabilizer of a symbol.

In this section, $\theta$ always means a homomorphism of $\pi K$ on $A_{5}$. Since the overgenerators of $\pi K$ in any normal presentation (1) are all conjugate, the elements $x_{i} \theta \in A_{5}$ all have the same order, say $r$. If $y_{1}$ is a generator in a second normal presentation of $\pi K$, then $y_{1}$ is conjugate to $x_{1}$ or $x_{1}^{-1}$, therefore $y_{1} \theta$ has order $r$. This means that the number $r$ depends only on $\theta$ and not on the presentation, and this allows us to define the order of $\theta$ as $r$. Equivalent homomorphisms obviously have the same order, so that the problem of classifying the various homomorphisms is split into three cases. From now on, we assume that the number of generators $n$ in the normal presentation (1) is 2 or 3 .

If $\theta$ has order 5 , then $x_{1} \theta$ is a 5-cycle in $A_{5}$. For some $\alpha \in S_{5}, \alpha^{-1}\left(x_{1} \theta\right) \alpha=$ $12345=C$ so that $\theta$ is equivalent to a homomorphism $\theta_{1}$ such that $x_{1} \theta_{1}=C$. We may assume $\theta=\theta_{1}$. Next, $x_{2} \theta$ is one of the 12 elements of $A_{5}$ conjugate to $C$. These are $C, C^{-1}$, and 10 other elements which do not commute with $C$. These 10 elements lie in two sets, each of 5 elements, on which conjugation by $C$ is a cyclic permutation. If one of these elements is $\alpha$, then $\left\{C^{-i} \alpha C^{j} \mid j=0, \cdots, 4\right\}$ is one set and $\left\{C^{-j} \alpha^{-1} C^{j} \mid j=0, \cdots, 4\right\}$ is the other. Thus, $x_{2} \theta=C, C^{-1}$, or the automorphism of $A_{5}$, defined by conjugation by a suitable power of $C$, carries $x_{2} \theta$ to $\alpha$ or $\alpha^{-1}$. Hence, we may assume $x_{2} \theta=C, C^{-1}, \alpha$ or $\alpha^{-1}$. No automorphism of $A_{5}$ that leaves $C$ fixed carries $\alpha$ to $\alpha^{-1}$, so that two homomorphisms $\theta_{1}, \theta_{2}$, thus normalized with $x_{2} \theta_{1} \neq x_{2} \theta_{2}$, are inequivalent. If $n=2$, then $\left\langle C, x_{2} \theta\right\rangle=A_{5}$ so that $x_{2} \theta=\alpha$ or $\alpha^{-1}$. We shall fix the element $\alpha$ to be (13254) $=$ [35214].

Suppose $n=3$ and $x_{2} \theta=C^{ \pm 1}$. We can apply the above argument to $x_{3} \theta$ and get $x_{3} \theta=\alpha$ or $\alpha^{-1}$. However, if $x_{2} \theta=\alpha^{ \pm 1}$ then $\left\langle C, x_{2} \theta\right\rangle=A_{5}$ so that the only automorphism which leaves $x_{1} \theta$ and $x_{2} \theta$ fixed is the identity. This means that $x_{3} \theta$ can be any of the 12 elements conjugate to $C$ and that two homomorphisms normalized so $x_{1} \theta_{1}=x_{1} \theta_{2}=C, x_{2} \theta_{1}=x_{2} \theta_{2}=\alpha^{ \pm 1}$, but $x_{3} \theta_{1} \neq x_{3} \theta_{2}$, are inequivalent. Hence, when $n=3$ the original homomorphism $\theta$ is equivalent to a homomorphism defined by one of $2 \cdot 2+2 \cdot 12=28$ choices for the images of $x_{1}, x_{2}, x_{3}$.

Now let $\theta$ be a homomorphism of order 3. We may assume $x_{1} \theta=123$. We have two cases, either 123 and $x_{2} \theta$ generate $A_{5}$, or they do not. If they do, then $x_{2} \theta$ cannot leave 4 or 5 fixed and therefore $x_{2} \theta=a 45$ or $a 54$ where $a=1,2$ or 3 . When $x_{2} \theta=a 54$, the automorphism defined by (45) $\in S_{5}$ leaves 123 fixed and transforms $a 54$ to $a 45$, so that $\theta$ may be replaced by an equivalent homomorphism (still called) $\theta$ where $x_{2} \theta=a 45$. If $\alpha$ is a suitable power of 123 , then $a 45^{\alpha}=145$ so that we may finally assume $x_{2} \theta=145$. Note that $\langle 123,145\rangle=A_{5}$ and that this argument shows that if $\beta$ is a 3 -cycle moving both 4 and 5 then $\langle 123, \beta\rangle=A_{5}$.

When $n=2$ we must have $\left\langle x_{1} \theta, x_{2} \theta\right\rangle=A_{5}$ so that the normalized homomorphism can only be $x_{1} \theta=123, x_{2} \theta=145$. However, when $n=3$ and $\theta_{1}, \theta_{2}$ are two homomorphisms thus normalized, then $\theta_{1} \equiv \theta_{2} \Leftrightarrow x_{3} \theta_{1}=x_{3} \theta_{2}$. Therefore, our original $\theta$ is equivalent to a homomorphism defined by one of 20 choices for the images of $x_{1}, x_{2}, x_{3}$ when $\left\langle x_{1} \theta, x_{2} \theta\right\rangle=A_{5}$.

Next, suppose that $\theta$ is a homomorphism of order 3 such that $x_{1} \theta=123$ and $\langle 123\rangle<\left\langle x_{1} \theta, x_{2} \theta\right\rangle<A_{5}$. Then $x_{2} \theta$ must move at least one of 4,5 , but not both. 
If $x_{2} \theta$ moves 5, the automorphism defined by (45) $\in S_{5}$ transforms $\theta$ to an equivalent homomorphism such that $x_{2} \theta$ moves 4 , therefore, we can assume $x_{2} \theta$ moves 4 . Now $x_{2} \theta=a b 4$ where $a$ and $b$ are $1,2,3$. We wish to normalize $\theta$ so that $a=1$, in which event $x_{2} \theta=124$ or 134. If $a \neq 1$ already, the appropriate automorphism is defined by $1 a c \in S_{5}$ where $c=2$ or 3 as $a=3$ or 2 . It is easy to check that if $x_{1} \theta_{1}=x_{2} \theta_{2}=$ $123, x_{2} \theta_{1}=124, x_{2} \theta_{2}=134$, then $\theta_{1} \not \equiv \theta_{2}$. Furthermore, $\langle 123,124\rangle=\langle 123,134\rangle=$ the subgroup of $A_{5}$ leaving 5 fixed. This subgroup is maximal so that if $\zeta$ is a 3-cycle moving 5 then $\left\langle x_{1} \theta, x_{2} \theta, \zeta\right\rangle=A_{5}$. When $n=3, x_{3} \theta$ must be one of these 123 -cycles $\zeta=a b 5$. There is no freedom in the choice of $\theta$ in its equivalence class left to move $x_{3} \theta$, so that two homomorphisms $\theta_{1}, \theta_{2}$ normalized so that $x_{1} \theta_{1}=x_{2} \theta_{2}=123$, $x_{2} \theta_{1}=x_{2} \theta_{2}=124$ or 134 , but $x_{3} \theta_{1} \neq x_{3} \theta_{2}$, are inequivalent. Therefore, our original $\theta$ is equivalent to a homomorphism defined by one of $2 \cdot 12=24$ choices for the images of $x_{1}, x_{2}, x_{3}$ when $n=3, \theta$ has order 3 , and $\left\langle x_{1} \theta\right\rangle\left\langle\left\langle x_{1} \theta, x_{2} \theta\right\rangle\left\langle A_{5}\right.\right.$.

Finally for order 3 assume that $x_{2} \theta=123$ or 132 . Then $\left\langle 123, x_{3} \theta\right\rangle=A_{5}$ and the normalization argument above shows that we may replace $\theta$ by a new $\theta$ such that $x_{3} \theta=145$. Our original $\theta$ is equivalent to a homomorphism defined by one of 2 choices for the images of $x_{1}, x_{2}, x_{3}$.

Last and least we consider homomorphisms of order 2. It is well known (and easy to check for $A_{5}$ ) that a nonabelian group generated by two elements of order 2 is dihedral, hence such homomorphisms do not exist for $n=2$. We omit the case by case argument for $n=3$ and simply tabulate the results in Table 1 at the end which summarizes the discussion of this section.

Now that we have a normal form for each equivalence class of homomorphisms we can test for the existence of each equivalence class of homomorphisms by testing the assignments $x_{1} \theta, \cdots, x_{\nu} \theta$ of its normal form to see if $\theta$ actually defines a homomorphism, as remarked in the introduction.

3. We now generalize in a naive manner some of the results of the last section to a classification of homomorphisms of knot groups on the linear projective groups $L_{p} \equiv L F(2, p) \equiv P S L(2, p)$ where $p$ is a rational prime. For $L_{5}=A_{5}$, we found that the easiest case to handle was the homomorphisms of order 5 . Accordingly, we shall fix our attention on the homomorphisms of order $p$ on $L_{p}$, and for brevity, we shall call such a homomorphism a "rep". We refer to Chapter XIV of Burnside [1] for the relevant background. The group $L_{2}$ is somewhat exceptional so we shall assume $p$ is an odd prime in the classification arguments that follow.

The group $L_{p}$ has order $p \cdot\left(p^{2}-1\right) / 2$ and is simple except when $p=3$ where $L_{3} \approx A_{4}$. It contains $p^{2}-1$ elements of order $p$ and these lie in two conjugacy classes. If $\alpha \in L_{p}$ has order $p$, then $\alpha$ is conjugate to $\alpha^{n}$ iff $n$ is a square $(\bmod p)$. If $\beta$ also has order $p$, either $\beta=\alpha^{n}$ for some $n$ or $\langle\alpha, \beta\rangle=L_{p}$. All elements of order $p$ are either powers of $\alpha$ or lie in one of $p-1$ orbits of $p$ elements under conjugation by $\alpha$. The only automorphisms of $L_{p}$ that leave $\alpha$ fixed are conjugations by powers of $\alpha$. For any pair $\beta_{1}, \beta_{2}$ of elements of order $p$ there is an automorphism $\zeta$ of $L_{p}$ such that $\beta_{2}=\beta_{1} \zeta$.

Let (1) be a normal presentation of the knot group $\pi K$ and assume the number of generators $n$ is 2 or 3 . To begin the classifications of the reps of $\pi K$ on $L_{p}$, we fix $\alpha \in L_{p}$ of order $p$ and alter each rep by an automorphism of $L_{p}$ so that the image of $x_{1}$ is $\alpha$. Next, consider the $(p-1) / 2$ orbits of elements of order $p$ which are conjugate 
to $\alpha$. If we choose an element $\alpha_{j}$ in the $j$ th of these for $j=1, \cdots,(p-1) / 2$, then every element $\beta$ of order $p$ which is not a power of $\alpha$ has the form $\beta=\alpha^{-s} \alpha_{i} \alpha^{8}$ for some $j$ and some $s$. This means that every rep $\theta$ can be altered so that $x_{1} \theta=\alpha$, $x_{2} \theta=\alpha^{8}$ for some $s$ or $x_{2} \theta=\alpha_{i}$ for some $j$. When $n=2$ the equivalence class of $\theta$ is completely determined by $j$.

When $n=3$, we have two cases. If $x_{2} \theta=\alpha_{i}$ for some $j$, then $\left\langle x_{1} \theta, x_{2} \theta\right\rangle=L_{p}$ so that the equivalence class of $\theta$ is completely determined by $j$ and $x_{3} \theta$. However, if $x_{2} \theta=\alpha^{8}$ for some $s,(s / p)=1$, then we can alter $\theta$ by an automorphism of $L_{p}$ so that $x_{3} \theta=\alpha_{i}$ for some $j$. In this event, the equivalence class of $\theta$ is determined by $s$ and $j$.

This shows that when $n=2$ the equivalence classes of reps can be found by $(p-1) / 2$ experiments and when $n=3$ by

$$
\frac{p-1}{2} \cdot \frac{p^{2}-1}{2}+\left(\frac{p-1}{2}\right)^{2}=(p+2)\left(\frac{p-1}{2}\right)^{2}
$$

experiments. The method generalizes immediately to $n>3$ generators and the general formula for the number of experiments is

$$
\left(\frac{p-1}{2}\right)^{n-1} \frac{(p+1)^{n-1}-1}{p}
$$

We can determine the existence of reps on the solvable groups $L_{2}$ and $L_{3}$ from the Alexander polynomial $\Delta_{K}(x)$ of $K$. Since $L_{2}$ is dihedral of order 6 , the argument of Section 10 of [6] shows that $\pi K$ has a rep on $L_{2}$ iff 3 divides $\Delta_{K}(-1)$. By a variation of this argument, we can show $\pi K$ has a rep on $L_{3}$ iff 2 divides $\Delta_{K}(\omega) \Delta_{K}(\bar{\omega})$ where $\omega$ is a nonreal cube root of 1 . (This strange number is the product of the "Torsionszahlen dritter Stufe" in Reidemeister [13] which are tabulated there on p. 25 for the classical knots. We note that his table is wrong for the knots $9_{1}$ and $9_{\theta}$, the entries in both cases under $h=3$ should read 2,2. The rest of his table is correct.)

As a first hint that there is a better way to find reps, we will show that some knots have a rep on $L_{p}$ for every prime $p$. Let $M$ be the group $S L(2, Z)$, then every $L_{p}$ is a homomorphic image of $M$. Furthermore, the image of $\left(\begin{array}{ll}1 & 1 \\ 0 & 1\end{array}\right)$ in $L_{p}$ is an element of order $p$. This means that it is sufficient to find a homomorphism $\theta$ of $\pi K$ on $M$ such that $x_{1} \theta=\left(\begin{array}{ll}1 & 1 \\ 0 & 1\end{array}\right)$. The elements $A=\left(\begin{array}{ll}1 & 1 \\ 0 & 1\end{array}\right)$ and $B=\left(\begin{array}{rr}1 & 0 \\ -1 & 1\end{array}\right)$ are conjugate and generate $M$, in fact $B A B=C=\left(\begin{array}{rr}0 & 1 \\ -1 & 0\end{array}\right), A B=\left(\begin{array}{rr}0 & 1 \\ -1 & 1\end{array}\right)=D$, and we know from Section 1.4, Exercise 19, of [11], that $M=\langle C, D\rangle$. Using the presentations in Table 2, we find the following homomorphisms $\theta$ of $\pi K$ on $M$ such that $x_{1} \theta=\left(\begin{array}{ll}1 & 1 \\ 0 & 1\end{array}\right)$.

$$
\begin{array}{ccc}
K & x_{2} \theta & x_{3} \theta \\
3_{1}, 9_{1}, 9_{6}, 9_{23} \quad(\nu=2) & \left(\begin{array}{rr}
1 & 0 \\
-1 & 1
\end{array}\right) & \\
8_{5}, 8_{18}, 9_{16} & \left(\begin{array}{rr}
1 & 0 \\
-1 & 1
\end{array}\right) & \left(\begin{array}{ll}
1 & 1 \\
0 & 1
\end{array}\right) \\
8_{18}, 8_{21}, 9_{24} & \left(\begin{array}{ll}
1 & 1 \\
0 & 1
\end{array}\right) & \left(\begin{array}{rr}
1 & 0 \\
-1 & 1
\end{array}\right)
\end{array}
$$

(continued) 


$$
\begin{aligned}
& \begin{array}{lll}
x_{2} \theta & x_{3} \theta
\end{array} \\
& 8_{20} \quad\left(\begin{array}{rr}
1 & 0 \\
-1 & 1
\end{array}\right) \quad\left(\begin{array}{rr}
1 & 0 \\
-1 & 1
\end{array}\right) \\
& 8_{18}, 9_{28} \quad\left(\begin{array}{rr}
1 & 0 \\
-1 & 1
\end{array}\right) \quad\left(\begin{array}{rr}
3 & 4 \\
-1 & -1
\end{array}\right) \\
& 8_{10}, 8_{15}, 8_{18}, 9_{40} \quad\left(\begin{array}{rr}
1 & 0 \\
-1 & 1
\end{array}\right) \quad\left(\begin{array}{rr}
2 & 1 \\
-1 & 0
\end{array}\right) \\
& 8_{19} \quad\left(\begin{array}{rr}
1 & 0 \\
-1 & 1
\end{array}\right) \quad\left(\begin{array}{rr}
0 & 1 \\
-1 & 2
\end{array}\right)
\end{aligned}
$$

The above list is complete up to equivalence for the classical knots. Because a necessary condition for such a homomorphism $\theta$ on $M$ is the existence of a rep on $L_{p}$ for every $p$, the nonexistence of other $\theta$ follows from the above mentioned table in Reidemeister or from Table 3 in the microfiche section in this issue.

4. If (1) is a normal presentation of $\pi K$ on a small number $n$ of generators, the relators are frequently very long words in $x_{1}, \cdots, x_{n}$. For example, for some rational knots with 9 crossings, the relator is a word in $x_{1}, x_{2}$ of length $>100$. This difficulty can be avoided by introducing new generators $x_{n+1}, \cdots, x_{m}$, and new relations expressing $x_{n+i}$ as a word in $x_{1}, \cdots, x_{n+i-1}$, for $i=n+1, \cdots, m$. The resulting presentation of $\pi K$ now has the form:

$$
\begin{array}{ll}
\text { generators } & x_{1}, \cdots, x_{m}, \\
\text { relations } & x_{n+i}=\text { word in } x_{1}, \cdots, x_{n+i-1}, i=1, \cdots, m-n, \\
\text { relators } & r_{1}\left(x_{1}, \cdots, x_{m}\right), \cdots, r_{n-1}\left(x_{1}, \cdots, x_{m}\right) .
\end{array}
$$

This is still a normal presentation on $n$ generators $x_{1}, \cdots, x_{n}$ and much more convenient in practice. Note that the subordinate generators $x_{n+1}, \cdots, x_{m}$ are arbitrary elements of $\pi K$ and not necessarily over generators of $\pi K$.

If one is given a model knot $k$ for $K$, a presentation (3) is defined implicitly by orienting $k$ and selecting $n$ arcs of the picture to correspond to $x_{1}, \cdots, x_{n}$. Next, the other arcs are numbered $n+1, \cdots, m$ in such a way that at one end $\operatorname{arc}(j)$ is crossed by an $\operatorname{arc}(i)$ with $i<j$ and on the other side of $\operatorname{arc}(i)$ the continuation of $\operatorname{arc}(i)$ is $\operatorname{arc}(s)$ with $s<j, j=n+1, \cdots, m$. (This rule is stated for the Wirtinger presentation, but for an over-presentation for a nonalternating knot the rule is only slightly more complex. See the example below.)

$$
\begin{aligned}
& \text { Presentation } \pi K=\left|x_{1}, x_{2}, x_{3}: r_{1}, r_{2}\right| \\
& x_{4}=x_{3}^{-1} x_{2} x_{3}, \\
& x_{5}=x_{3}^{-1} x_{2}^{-1} x_{1} x_{2} x_{3} \text {, } \\
& r_{1}=x_{5} x_{1} x_{2}^{-1} x_{1}^{-1} \text {, } \\
& r_{2}=\begin{array}{llllll}
x_{4} & x_{1} & x_{2} & x_{3}^{-1} & x_{2}^{-1} & x_{1}^{-1}
\end{array}
\end{aligned}
$$

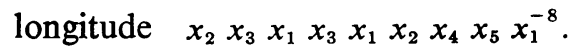




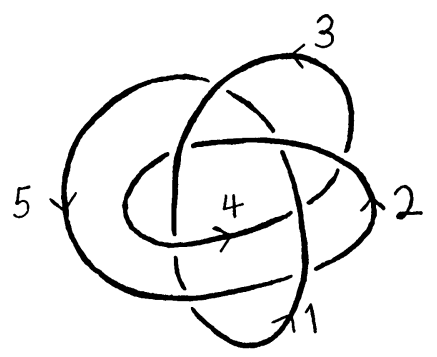

FIGURE 1

Table 2 in the microfiche section of this issue is a list of normal presentations of the marked groups of the classical knots. These presentations have been checked in numerous ways and are almost certainly correct. Table 3 of homomorphisms of these knot groups on $A_{5}$ is based on Table 2.

5. Associated with a homomorphism $\theta$ of a knot group $\pi K$ on a finite group $G$ are certain topological spaces whose homotopy types are invariants of the knot type $K$. In particular, suppose $G$ is a transitive group of permutations of the symbols $1, \cdots, s$, and let $H$ be the subgroup of $\pi K$ whose image in $G$ keeps the symbol 1 fixed. Let $\mathcal{U}$ be the covering space of $S^{3}-k$ corresponding to the subgroup $H$, then $\mathcal{U}$ is a noncompact $s$-sheeted covering manifold of $S^{3}-k$. Furthermore, as explained in Section 8 of [6], $u$ has a certain natural closure $\mathfrak{T}$ which is a compact manifold known as the $s$-sheeted cover of $S^{3}$ branched over $k$ (belonging to $H$ ). The spaces $\mathcal{U}, \mathfrak{T}$ are the topological spaces in question, they depend (up to homeomorphism) only on $\pi K$, the permutation representation of the group $G$, and the weak equivalence class of $\theta$. In particular, their integral homology groups $H_{1} \mathcal{U} \equiv H_{1}(\mathcal{U} ; Z), H_{1} \Re \mathcal{I} \equiv$ $\left.H_{1}(\Re) Z Z\right)$ are invariants of $K$. A general algorithm to compute integral presentation matrices for these homology groups is informally derived in Section 8 of [6] as a consequence of a presentation for the fundamental groups $\pi_{1} \mathcal{U}, \pi_{1} \mathfrak{T}$.

We have translated this algorithm into a Fortran subroutine, Homology, which goes into action given a presentation (2) and the images of the generators $\left\{x_{i} \theta, i=\right.$ $1, \cdots, n\}$ expressed as permutations. It returns the groups $H_{1} \mathcal{U}, H_{1} \Re($ as Betti numbers and torsion coefficients. The subroutine has the same generality as the original algorithm (except for practical limitations) and so can be used to study more general groups than knot groups. A more complete description and a listing of Homology have been deposited in the UMT file; cf. the review in the review section of this issue.

To apply Homology to a homomorphism $\theta$ of $\pi K$ on $L_{p}, p$ prime, we need a permutation representation for $L_{p}$. The different permutation representations of $L_{p}$ will give rise to different spaces $\mathcal{U}, \mathfrak{T}$, but the smaller the number of sheets $s$, the smaller the presentation matrices for $H_{1} \mathcal{U}, H_{1}$ IT. It is known ([1] or [9]) that $L_{p}$ always has a permutation representation of degree $p+1$ but none of lower degree, except for $p=5,7,11$, where there are representations of degree $p$. So for all $p \neq 5$, 7,11 , we restrict our attention to the representation of degree $p+1$ but for $p=5,7$, 11 we shall use both representations. When $\pi K, p$ and $\theta$ are understood, we shall write $\mathfrak{U}_{p+1}, \mathfrak{T}_{p+1}, \mathcal{U}_{p}, \mathfrak{T}_{p}$ for the corresponding covering spaces. 
For the exceptional primes $5,7,11$, we took particular permutation groups of degree $p$ for the groups $L_{p}$. For $p=5$, we used $A_{5}$ as discussed in Section 2. For $p=7$, we took the elements $\alpha, \alpha_{1}, \alpha_{2}, \alpha_{3}$ of Section 3 to be

$$
\alpha=(1234567), \quad \alpha_{1}=(1675243), \quad \alpha_{2}=\alpha_{1}^{2}, \quad \alpha_{3}=\alpha_{1}^{4} .
$$

This representation of $L_{7}$ is taken from p. 208, line 5 of [1]. We took the elements $\alpha,\left\{\alpha_{i}, j=1, \cdots, 5\right\}$ for $L_{11}$ to be

$\alpha=(123456789 a b), \quad \beta=(1 a)(37)(56)(89)(2)(b), \quad \alpha_{i}=\beta \alpha^{i^{2}} \beta, \quad j=1, \cdots, 5$.

This representation came from 7.8 of [3]. In addition, for $p=5$, 7, we wrote out specific isomorphisms between the above permutation groups and permutation groups of degrees 6,8 resp. We may someday do the same for $p=11$.

For the nonexceptional primes $p$, we find it convenient to deal with the groups $L_{p}$ directly as matrix groups and derive our permutations from the matrices. Our work in this direction is not complete except for the primes $p \leqq 19$.

Given the permutation group $G$ of degree $s$ and the homomorphism $\theta$ of $\pi K$ on $G$, suppose that $x_{1} \theta$ has $c$ disjoint cycles. Then, as Professor Fox shows, the number of generators for $\pi_{1} \Re\left(\right.$ (and consequently for $H_{1} \Re$ ) is bounded above by $n s-n c-$ $(s-1)$; cf. [7]. When this bound is $0, \mathfrak{T}$ is a homotopy sphere. When $\nu K=2$ this is true for $\Re_{3}$ when $\theta$ is a rep on $L_{2}$ [7] and for $\Re_{5}$ when $\theta$ is a homomorphism of order 3 on $A_{5}$. Consequently, we do not compute $H_{1} u, H_{1} \Re$ in these cases. When the upper bound is $1, \pi_{1} \Re C=H_{1} \Re$ is cyclic and this happens for $G=L_{2}, \nu K=3$, and for $G=L_{3}, \nu K=2$. (The number $c$ is always 2 for the permutation representation of $L_{p}$ of degree $p+1$.)

The space $U$ can be considered as the complementary space of the link $l=\Re-U$ in $\Re$. The link type of $l$ in $\Re$ is an invariant of the knot type $K$ and the group $H_{1} u$ is only the crudest of a set of link invariants of $l$ analogous to the Alexander polynomials and the Hosokawa polynomial [8]. We have not pursued this matter beyond the calculation (by hand) of one specific example. The rational knot 5 has a homomorphism of degree 3 on $A_{5}$ (Table 3), consequently $\mathfrak{M}_{5}$ is a homotopy sphere and $l$ is a link in this sphere. We found that the Alexander matrix of $l$ (in one variable $x$ ) reduces to

$$
a(x)=\left\|\begin{array}{cc}
\alpha \beta & 0 \\
0 & \alpha \beta \gamma
\end{array}\right\|,
$$

where $\alpha=1-x, \beta=1+x^{5}$ and $\gamma=1+x+x^{2}+x^{3}+x^{4}$. Consequently, the Hosokawa polynomial is $\nabla(x)=\beta^{2} \gamma$.

6. The algorithms of the preceding sections have been completely or partially coded as Fortran programs and put to work on up to 250 knots. In this and the next section we consider the results. They fill quite a few tables, some rather lengthy, so we will include just one in microfiche and merely discuss the conclusions to be drawn from the rest.

In this and the next section, we describe a homology group $H_{1} X \equiv H_{1}(X ; Z)$ by the diagonal entries of a square presentation matrix for $H_{1} X$. If convenient, some of these entries may be 1 . When the coefficient group is not $Z$, it will be $Z_{n}=Z / n Z$ for 
some $n$. For example, $H_{1} X=0,1,3$ means $H_{1} X=\mathrm{Z} \oplus Z_{3}$, and if $H_{1} X=3$ then $H_{1}\left(X ; Z_{2}\right)=1$.

We begin with the reps on $L_{2}$. After this paper was submitted for publication, the paper "Metacyclic invariants of knots and links" by R. H. Fox appeared in Canad. J. Math., v. 22, 1970, pp. 193-201, and parts of that paper are very relevant to our work. In the first place, Fox points out that the longitudes of a knot group $\pi K$ lie in the second commutator subgroup of $\pi K$, hence if $\theta: \pi K \rightarrow G$ where $G$ is a group whose second commutator subgroup is trivial, the longitudes are in the kernel of $\theta$. This applies when $G=L_{2}$ or $L_{3}$ and supersedes a more cumbersome argument of mine. Secondly, Fox includes a table (which agrees with mine) of the groups $H_{1} \mathfrak{M T}_{3}$ associated with the reps of the groups of the classical knots on $L_{2}$. More interesting is the linking number $v$ of the two components of $\mathfrak{N}_{3}-\mathfrak{U}_{3}$ for each rep which Fox includes in his table. Although I do not know how to calculate $v$, I found on comparison with one of my tables that in certain cases there seems to be a strict relation between $v$ and a number I can calculate. Namely, if $\theta$ is a rep of $\pi K$ on $M=S L(2, Z)$ where $x_{1} \theta=\left(\begin{array}{ll}1 & 1 \\ 0 & 1\end{array}\right)$, and $\gamma \in \pi K$ is a longitude commuting with $x_{1}$, then $\gamma \theta$ necessarily has the form $\pm\left(\begin{array}{ll}1 & o \\ 0 & 1\end{array}\right)$ for some $g \in Z$. In fact, $g \equiv 0(\bmod 6)$ because the image of $\gamma$ in $L_{2}$ or $L_{3}$ is $E$. Then, in the 15 cases where Fox's homomorphism is the mod 2 reduction of such a homomorphism on $M$, we have

$$
g= \pm 3 v \text {. }
$$

The sign depends on the normalizations and we cannot settle it here. This relation is probably at the heart of the reason why in all known cases $v$ is a rational fraction with an even numerator.

For $A_{5}=L_{5}$, we tabulate the detailed results for the classical knots in Table 3 in microfiche. All permutations in that table are in the bracket [ ] notation. There are altogether 51 reps, 26 homomorphisms of order 3, and 9 of order 2, for a total of 86 homomorphisms. There are 30 knots with no homomorphism of which only $9_{32}, 9_{33}, 9_{34}$ are not rational. Five knots, viz. $9_{16}, 9_{22}, 9_{25}, 9_{30}, 9_{36}$, are proven nonrational by the number or types of their homomorphisms but not by any simpler method that I know. Most of the nonamphicheiral knots are proven to be such by Fox's argument [5].

The cases $p=7$ and $p=11$ have been run on the computer for the classical knots. We found 102 reps on $L_{7}$ for these knots and that 27 knots have no rep, of which 6 are not rational. The maximal number of reps for any knot is 13 for $9_{40}$ and these appear to lie in 6 weak equivalence classes. There are 100 reps on $L_{11}$ and 29 knots with no reps, 8 of these are not rational. This time the maximal number of reps per knot is only 5 , attained for $K=9_{41}, 9_{47}$ and $9_{48}$.

This is as far as we have gone for the block of classical knots and as far as we have computed the homology. We have gone further to find at least one rep on some $L_{p}$ for every classical knot. The largest prime needed for the first rep was 23 which was required for $7_{5}$ and $8_{12}$. All the nonrational knots, save $9_{33}$, have a rep on $L_{p}$ for $p \leqq 11$, but for $9_{33}$ we need $p=17$.

Out of the detailed results of the calculation of around 1000 homology groups $H_{1} \mathcal{U}_{h}, H_{1} \Re_{h}$, we have found three general conjectures that appear certain, and two more which are very likely. The discussion splits naturally into the cases $h=p+1$, $h=p$, and we exclude $p=2$ because 2 is quite exceptional in this context. As 
notation, write $B\left(\mathcal{U}_{h}\right), B\left(\mathfrak{T} \mathcal{T}_{h}\right)$ for the Betti numbers of $\mathcal{U}_{h}, \mathfrak{T}_{h}$, respectively. We begin with $h=p+1$.

Conjecture A. If $\theta$ is a rep and $p \geqq 3$,

$$
B\left(\mathfrak{T U}_{p+1}\right)+2 \geqq B\left(\mathcal{U}_{p+1}\right) \geqq B\left(\mathfrak{T U}_{p+1}\right)+1,
$$

and when $B\left(\mathfrak{T T}_{p+1}\right)=0, B\left(\mathcal{U}_{p+1}\right)=2$. Furthermore $H_{1} \mathfrak{T}_{p+1}$ has a torsion generator of even order.

The usual value for $B\left(\Re_{p+1}\right)$ is zero. We know that $H_{1} \Re_{p+1}$ is a factor group of $H_{1} \mathcal{u}_{p+1}$, but apart from that and Conjecture A there seems to be no further universal assertion to be made about the comparison of $H_{1} u_{p+1}$ with $H_{1} \Re_{p+1}$. However, when $\theta$ happens to be the reduction of a homomorphism on $M$ the situation is much clearer. We have:

Conjecture B. For each prime $p \geqq 2$, there are integers $b_{p}$ and $d_{p}$, where $d_{p}$ is a divisor of $p+1$, which have the following properties. Let $\theta: \pi K \rightarrow L_{p}$ be the reduction modulo $p$ of a homomorphism $\phi: \pi K \rightarrow S L(2, Z)$ such that $x_{1} \phi=\left(\begin{array}{ll}1 & 1 \\ 0 & 1\end{array}\right)$. Let the torsion subgroups of the corresponding homology groups $H_{1} \varkappa_{p+1}, H_{1} \Re_{p+1}$ have orders $u, m$, respectively. Then, $B\left(\Re_{p+1}\right) \geqq b_{p}$ and $m / u$ is an integer which divides $d_{p}$. If $B\left(\Re_{p+1}\right)=b_{p}$, then $m / u=d_{p}$.

We have verified this when $p \leqq 19$ for the homomorphisms on $M$ listed in Section 3. The numbers $b_{p}, d_{p}$ for $p \leqq 19$ are

$\begin{array}{rrrrrrrrr}p & 2 & 3 & 5 & 7 & 11 & 13 & 17 & 19 \\ b_{p} & 0 & 0 & 0 & 0 & 2 & 0 & 2 & 2 \\ d_{p} & 1 & 2 & 2 & 4 & 2 & 14 & 6 & 10\end{array}$

It should be emphasized that Conjecture B only concerns the orders $u, m$ of the torsion subgroups, not the relations between the actual torsion coefficients. The tables show that the passage from $\mathcal{U}$ to $\Re($ will do the wildest things to these torsion numbers, subject to the known or conjectured restrictions. We have also considered the question of patterns in the groups $H_{1} \mathcal{U}_{p+1}, H_{1} \Re_{p+1}$ for a fixed $K$ and varying $p$ (corresponding to a homomorphism on $M$ ), but the results are too vague to be commented on here.

There is a conjecture similar to conjecture $A$ for the groups $H_{1} \mathcal{U}_{6}, H_{1} \mathfrak{F T}_{6}$ corresponding to a homomorphism of order 2 or 3 on $L_{5}=A_{5}$. For order 3 , the conjecture is the same as Conjecture $\mathrm{A}$, and for order 2 the only change is

$$
B\left(\mathscr{T K}_{6}\right)+1 \leqq B\left(\mathcal{U}_{6}\right) \leqq B\left(\mathscr{T T}_{6}\right)+4 .
$$

In all known cases, $B\left(\mathfrak{T T _ { B }}\right)=0, B\left(\mathcal{U}_{6}\right)=4$, but this is not a conjecture.

Now for the groups $H_{1} \varkappa_{p}, H_{1} \Re_{p}$ when $p=5,7$ or 11 . We only discuss the cases where $\theta$ is a rep because the other cases are very different.

Conjecture C. If $\theta: \pi K \rightarrow L_{p}$ is a rep and $p=5,7$ or 11 then $B\left(\mathcal{u}_{p}\right)=B\left(\mathfrak{I T} \tau_{p}\right)+1$. If $H_{1} u_{p}=0, a_{1}, \cdots, a_{n}$ with $1 \leqq a_{1} \leqq a_{2} \leqq \cdots \leqq a_{n}$ then for some $j \in\{1, \cdots, n\}$, $H_{1} \Re_{p}=a_{1}, \cdots, p a_{i}, \cdots, a_{n}$.

In Conjecture $\mathrm{C}$ the torsion number $a_{i}$ may or may not be already divisible by $p$. The hypothesis $B\left(\mathcal{U}_{p}\right)=1$ is essential.

The two minor conjectures are that if $H_{1} u_{p}=0, a_{1}, \cdots, a_{n}$ as in Conjecture C, then 3 divides $a_{n}$ when $p=5$ and 2 divides $a_{n}$ when $p=7$. The evidence for these is quite strong, but because there is no corresponding rule for $p=11$, we hesitate to 
be too dogmatic about them. As an example, the knot $7_{4}$ has a unique rep on $L_{11}$, and $H_{1} u_{11}=0, H_{1} \Re_{11}=11$.

To conclude this section, consider the three knots of Fig. 2, of interest because they have Alexander polynomial $\Delta(x) \equiv 1$. The knot $k_{1}$ is

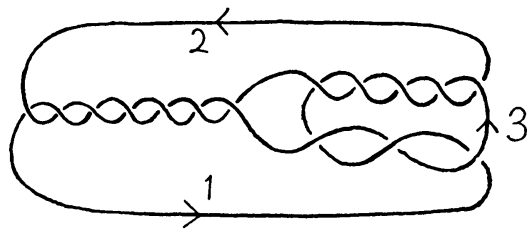

$k_{1}$

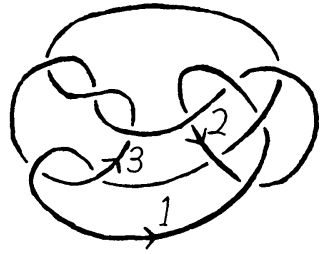

$k_{2}$

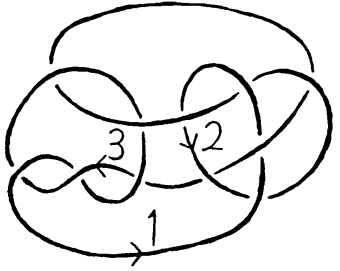

$k_{2}$

FIGURE 2

Seifert's classical example of such a nontrivial knot. The knot $k_{2}$ was constructed by Kinoshita and Terasaka [10] and further studied in [12]. The knot $k_{3}$ was discovered by J. Conway [2] in his classification of the nonalternating 11 crossing knots. He has shown that $k_{2}$ and $k_{3}$ are the only 11-crossing knots with $\Delta(x) \equiv 1$. (In Conway's notation, $k_{2}=.-(2,3) .2$ and $k_{3}=.-(3,2) .2$.) We first try the homomorphisms on $A_{5}$.

$$
\begin{aligned}
& K_{1} \quad x_{1} \theta=(12345), \quad x_{2} \theta=(15432), \quad x_{3} \theta=(14523) \text {. } \\
& H_{1} \mathfrak{U}_{5}=0,2,6 . \quad H_{1} \mathfrak{T}_{5}=2,30 . \quad H_{1} \mathfrak{U}_{6}=0,0,2,4 . \quad H_{1} \Re_{6}=2,2,16 . \\
& K_{2} \quad x_{1} \theta=(123), \quad x_{2} \theta=(145), \quad x_{3} \theta=(123), \quad \text { (longitude) } \theta=E \text {. } \\
& K_{3} \quad x_{1} \theta=(123), \quad x_{2} \theta=(145), \quad x_{3} \theta=(142), \quad \text { (longitude) } \theta=E \text {. }
\end{aligned}
$$

For both $K_{2}$ and $K_{3}$ :

$$
H_{1} \mathcal{U}_{5}=0,0,0 . \quad H_{1} \Re_{1}=7 . \quad H_{1} \mathfrak{U}_{6}=0,0,12 . \quad H_{1} \Re_{6}=180 .
$$

This shows that all three knots are nontrivial and that $K_{1}$ is different from the other two. We have not yet shown $K_{2} \neq K_{3}$. It is possible that the deeper Alexander invariants of the link $\Re(\mathcal{U}-\mathcal{U}$ in $\Re($ can do that, but I bet not.

We now try reps on $L_{7}$ for $K_{2}$ and $K_{3}$. For convenience, write $C=(1234567)$. Each rep satisfies $x_{1} \theta=C$ and each knot has two reps.

$$
\begin{aligned}
& K_{2}\left\{\begin{aligned}
x_{2} \theta_{1} & =(1675243), & x_{3} \theta_{1} & =(1452736), \quad \text { (longitude) } \theta_{1}=C . \\
H_{1} \mathfrak{u}_{7} & =0,4,28 . & H_{1} \Re_{7} & =28,28 . \quad H_{1} \mathfrak{U}_{8}=0,0,3 . \quad H_{1} \Re_{8}=1920 . \\
x_{2} \theta_{2} & =C, & x_{3} \theta_{2} & =(1675243), \quad \text { (longitude) } \theta_{2}=C^{2} . \\
H_{1} \mathfrak{U}_{7} & =0,2,238 . & H_{1} \Re_{7} & =14,238 . \quad H_{1} \mathfrak{U}_{8}=0,0,6 . \quad H_{1} \Re_{8}=3,228 .
\end{aligned}\right.
\end{aligned}
$$

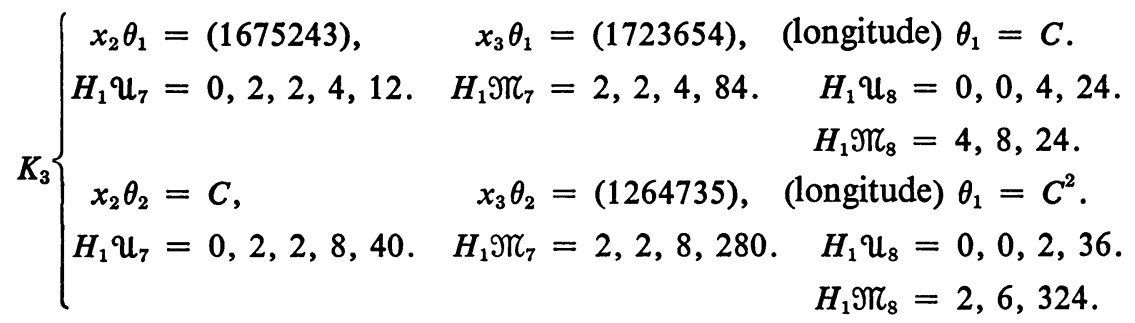


This shows that $K_{2} \neq K_{3}$ and that neither knot is amphicheiral. The results for $L_{11}$ are very similar: two reps per knot but the groups $H_{1} \mathfrak{u}_{11}, H_{1} \Re_{11}$ prove the knots are different.

We tried to prove that $K_{2} \neq K_{3}$ by counting the reps on $L_{p}$ for $p=13,17,19,23$, 29 and 31 to see if there was ever a difference between the corresponding numbers for $K_{2}, K_{3}$. (This experiment took more than $3 \frac{1}{4}$ hours on the Southampton computer.) The results suggest the $\pi K_{2}$ and $\pi K_{3}$ have the same number of reps on $L_{p}$ for every prime $p$ and that these knots cannot be distinguished this way. In [12], Magnus and Peluso proved that $\pi K_{2}$ has reps on $L_{p}$ for an infinity of $p$, but because they only netted a small proportion of the true set of reps their results cannot help us. We hope to return to this question in a later paper.

7. One of the best ways to understand the behavior of our knot invariants is to calculate them for a family of knots where the presentations of the knot groups depend on integral parameters. As a final collection of examples, we consider the rather complicated family of Kinoshita-Terasaka (KT) knots. The KT knot $k(p, n)$ is drawn in Fig. 3 in the case

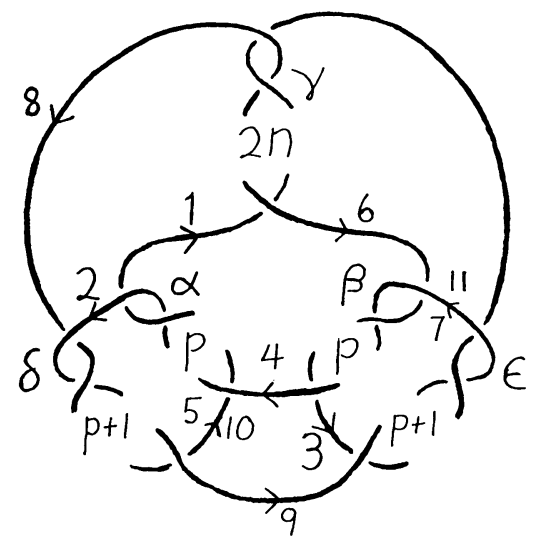

Figure 3. $\kappa(p, n)$

where $p$ and $n$ are positive integers. When $n<0$, the sense of rotation in the integral tangle (winding) $\gamma$ is reversed. In [10] Kinoshita and Terasaka proved that $\kappa(p, n)$ is a

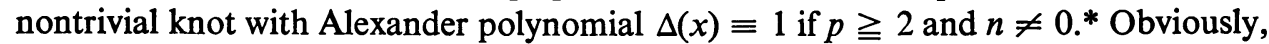
$\kappa(p, n)=0$ if $p=0$ or 1 or if $n=0$. The knot $K_{2}$ of the last section is $\kappa(2,1)$.

We may also define $k(p, n)$ for $p<0$ by reversing the sense of rotation in the tangles $\alpha, \beta, \delta, \epsilon$ in Fig. 3. (Beware that $\delta$ and $\epsilon$ now have $|p|-1=|(p+1)|$ crossings.) If we turn the knot over and reflect in a mirror, we see $\kappa(p, n)=\kappa(p,-n)$. Furthermore, we can turn the knot over and apply the Edmunds Flip to show $k(p, n)=$ $\kappa(-p-1, n)$ for all $p, n$. This allows us to assume $p$ is even and $n>0$ from now on.

Let $\pi(p, n)$ be the group of $\kappa(p, n)$. Since we have taken $p$ even, we may write $p=2 p_{0}, p_{+}=p_{0}+1$. Then $\pi(p, n)$ has a normal presentation (4) of the form $\left|x_{1}, x_{2}, x_{3}: r_{1}, r_{2}\right|$ where

\footnotetext{
* Our $\kappa(p, n)$ is written $\kappa(p, 2 n)$ in [10].
} 
(4)

$$
\begin{aligned}
& \alpha=x_{2} \quad x_{1} \quad x_{4}=\alpha^{p_{0}} x_{1} \alpha^{-p_{0}} \quad x_{5}=\alpha^{p_{0}} x_{2} \alpha^{-p_{0}} \\
& \beta=x_{4}^{-1} x_{3}^{-1} \quad x_{6}=\beta^{p_{0}} x_{4} \beta^{-p_{0}} \quad x_{7}=\beta^{p_{0}} x_{3} \beta^{-p_{0}} \\
& \gamma=x_{6} \quad x_{1}^{-1} \quad x_{8}=\gamma^{n} x_{1} \gamma^{-n} \\
& \delta=x_{2} x_{8} \quad x_{9}=\delta^{p_{0}} x_{2} \delta^{-p_{0}} \quad x_{10}=\delta^{p+} x_{8} \delta^{-p+} \\
& \epsilon=x_{9}^{-1} x_{3}^{-1} \quad x_{11}=\epsilon^{p_{0}} x_{9} \epsilon^{-p_{0}} \\
& r_{1}=x_{5} x_{10}^{-1} \quad r_{2}=x_{7} x_{11}^{-1}
\end{aligned}
$$

Suppose for some fixed $(p, n)$ that $\kappa(p, n)$ has a homomorphism $\theta$ of order $s$ on $A_{5}$. Say that the elements $\alpha \theta, \beta \theta, \gamma \theta, \delta \theta, \epsilon \theta$ of $A_{5}$ have orders $a, b, g, d, e$, respectively. Let $q$ be the 1.c.m. of $a, b, d, e$, and let $x_{i} \theta=\zeta_{i}, i=1,2,3$. From (4) it is easy to see that the assignment $x_{i} \phi=\zeta_{i}, i=1,2,3$, defines a homomorphism $\phi$ of $k\left(p^{\prime}, n^{\prime}\right)$ of order $s$ on $A_{5}$ whenever $p \equiv p^{\prime}(\bmod 2 q)$ and $n \equiv n^{\prime}(\bmod g)$. Because $\kappa(p, 0)=0$ we must have $g \geqq 2$, and because $\kappa(0, n)=\kappa(-2, n)=0$ we must have $2 q \geqq 6$ (in fact $2 q$ is 30 or 60 in every case). We shall say that the homomorphisms $\theta$ and $\phi$ are in the same clan of homomorphisms $\Theta$.

Because the numbers $g$ and $q$ for a clan of homomorphisms must be divisors of 30 , we know in advance that infinitely many KT knots have no homomorphisms on $A_{5}$. Clearly, the same difficulty will occur whatever finite (nonabelian) group $G$ we may use in place of $A_{5}$. Therefore, if we wish to resolve all the different types among the KT knots, we must either use an infinite group $G$ or an infinite set of finite groups $\left\{G_{n}, \nu=1,2, \cdots\right\}$.

We can show however, that infinitely many of the knots $\kappa(p, n)$ are different by considering the homology groups $H_{1} \mathfrak{U}_{h}, H_{1} \mathfrak{T}_{h}, h=5,6$, associated with a clan of homomorphisms $\Theta$. We examined a number of these clans and found by looking at 10 to 40 cases that these homology groups can usually be predicted by simple formulas. We must admit we have not tried to prove these formulas-the groups are complicated and the presentation matrices are rather large. However the method of proof (a giant calculation!) is clear and the results stated below should be convincing as they are. (Sceptics might try a simpler case of a clan $\Theta$ where the knots are alternating torus knots.) We present our formulas for five cases which illustrate most of what we should expect.

I. $x_{1} \theta=(123), x_{2} \theta=(123), x_{3} \theta=(145)$ when $p \equiv 2(\bmod 30), n \equiv 1(\bmod 3)$. Since $\kappa(p, n)=\kappa(p,-n)$, this clan also allows $n \equiv 2(\bmod 3)$, but using the standard normalization of $\theta$ in its equivalence class we then write it $x_{1} \theta=(123), x_{2} \theta=(145)$, $x_{3} \theta=(235)$. When $p=2$ and $n=3 s+1$ :

$$
H_{1} \mathfrak{U}_{5}=0,0,0 . \quad H_{1} \mathfrak{T U}_{5}=|7+24 s| . \quad H_{1} \mathfrak{U}_{6}=0,0,12 . \quad H_{1} \Re_{6}=|180+648 s| .
$$

II. $x_{1} \theta=(12345), x_{2} \theta=(13254), x_{3} \theta=(15324)$ when $p \equiv 2(\bmod 30)$, $n \equiv 2(\bmod 5)$. When $n \equiv 3(\bmod 5)$, we normalize $\theta$ to $x_{2} \theta=(14523), x_{3} \theta=(15324)$. (This clan is one of three clans of reps which exist for the same set of $(p, n)$.) When $p=2$ write $n=5 s+2, \tau=2$ if $11 \nmid s, \tau=22$ if $11 \mid s$,

$$
\begin{aligned}
& H_{1} \mathcal{U}_{5}=0,2,18 . \quad H_{1} \Re_{5}=2,90 . \quad H_{1} \mathcal{U}_{6}=0,0, \tau . \quad H_{1} \Re_{6}=|1100+2616 s| . \\
& \text { III. } x_{1} \theta=(12)(34), x_{2} \theta=(12)(35), x_{3} \theta=(13)(25) \quad \text { when } p \equiv 4(\bmod 30), \\
& n \equiv 1(\bmod 3) . \text { For } n \equiv 2(\bmod 3) \text { we write } x_{2} \theta=(13)(24), x_{3} \theta=(12)(35) . \text { When }
\end{aligned}
$$


$p=4, n=3 s+1$ and $\tau=|78+240 s|$ we get:

$$
H_{1} \mathfrak{U}_{5}=0,0,0, \tau . \quad H_{1} \Re_{5}=\tau . \quad H_{1} \mathfrak{U}_{6}=0,0,0,0,2 . \quad H_{1} \Re_{6}=4 .
$$

IV. $x_{1} \theta=(12)(34), x_{2} \theta=(13)(24), x_{3} \theta=(14)(25)$ when $p \equiv 2(\bmod 30)$, $n \equiv 2(\bmod 5)$. For $n \equiv 3(\bmod 5)$ we write $x_{3} \theta=(13)(25), x_{3} \theta=(15)(34)$. When $p=2$ the groups are:

$$
H_{1} \mathfrak{U}_{5}=0,0,0,3 . \quad H_{1} \Re_{5}=3 . \quad H_{1} \mathfrak{U}_{6}=0,0,0,0 . \quad H_{1} \Re_{6}=2 .
$$

V. $x_{1} \theta=(123), x_{2} \theta=(134), x_{3} \theta=(135)$ when $p \equiv 4(\bmod 60), n \equiv 1(\bmod 2)$. (This clan is one of four such which lie in pairs, the corresponding homology groups for the homomorphisms in a pair being the same.) When $p=4$ write $n=2 s+1$, $\rho=18|s+1|$, and let $\tau=9$ if $3 \mid s, \tau=3$ if $3 \nmid s$. Then:

$$
H_{1} \mathcal{U}_{5}=0,0,0, \tau . \quad H_{1} \mathfrak{T U}_{5}=2, \rho .
$$

$H_{1} \mathcal{U}_{6}=0,0, a(s), b(s)$ where $a(s)=4 \tau(s), b(s)=1$ when $s$ is even, $b(s)=4$ if $s \equiv 1(\bmod 4)$, and $b(s)=2$ if $s \equiv 3(\bmod 4) . H_{1} \mathfrak{M T}_{6}=c(s), d(s), t(s)$ where $c(s)=3$ if $s$ is even, $c(s)=2$, otherwise, and $d(s)=1$ if $s$ is even, $d(s)=3 \cdot b(s)$ otherwise. The final summand $t(s)$ varies so wildly that I cannot guess a formula for it.

Table 1. The images $x_{1} \theta, x_{2} \theta, x_{3} \theta$ of the generators of a normal presentation on three generators for the equivalence classes of homomorphisms on $A_{5}$. See Section 1 for the [ ] notation.

I. Order 5. $x_{1} \theta=[23451] .28$ combinations

$$
\begin{aligned}
x_{2} \theta= & {[35214] \text { or [43152]. } x_{3} \theta=[23451],[51234], \text { or one of } } \\
& {[35214],[41532],[54213],[31524],[54132], } \\
& {[43152],[25413],[43521],[24153],[35421] . } \\
x_{2} \theta= & {[23451] \text { or [51234]. } x_{3} \theta=[35214] \text { or [43152]. } }
\end{aligned}
$$

II. Order 3. $x_{1} \theta=[23145] .46$ combinations

$$
\begin{aligned}
& x_{2} \theta=[42351] . \quad x_{3} \theta=\text { any } 3 \text {-cycle. } \\
& x_{2} \theta=[24315] \text { or }[32415] . \quad x_{3} \theta=\text { any } 3 \text {-cycle which moves } 5 . \\
& x_{3} \theta=[23145] \text { or }[31245] . \quad x_{3} \theta=[42351] .
\end{aligned}
$$

III. Order 2. $x_{1} \theta=[21435] .19$ combinations

$$
\begin{array}{r}
x_{2} \theta=[21543] ., x_{3} \theta=\text { one of [35142], [32154], [34125], [42513], } \\
{[45312],[52431] .} \\
x_{2} \theta=[35142] ., x_{3} \theta=\text { one of [21543], [21354], [34125], [32154], } \\
{[43215],[45312],[53241],[52431],} \\
{[14523],[15432] .} \\
x_{2} \theta=[34125] . \\
x_{3} \theta=\text { one of [21543], [35142], [45312]. }
\end{array}
$$


1. W. S. BURNsIDE, Theory of Groups of Finite Order, Cambridge Univ. Press, 1897; 2nd ed., Dover, New York, 1955. MR 16, 1086.

2. J. H. ConwaY, An Enumeration of Knots and Links, and Some of Their Algebraic Properties, Computational Problems in Abstract Algebra, Pergamon Press, New York, 1970, pp. $329-358$.

3. H. S. M. CoXeter \& W. O. J. Moser, Generators and Relations for Discrete Group, Ergebnisse der Mathematik und ihrer Grenzgebiete, Band 14, Springer-Verlag, Berlin, 1965. MR 30 \#4818.

4. R. H. Crowell \& R. H. Fox, Introduction to Knot Theory, Ginn, Boston, Mass., 1963. MR 26 \#4348.

5. R. H. Fox, "On the complementary domains of a certain pair of inequivalent knots," Nederl. Akad. Wetensch. Proc. Ser. A., v. $55=$ Indag. Math., v. 14, 1952, pp. 37-40. MR 13, 966.

6. R. H. Fox, A Quick Trip Through Knot Theory, Topology of 3-Manifolds and Related Topics (Proc. Univ. of Georgia Inst., 1961), Prentice-Hall, Englewood Cliffs, N. J., 1962, pp. 120-167. MR 25 \#3522.

7. R. H. Fox, Construction of Simply Connected 3-Manifolds, Topology of 3-Manifolds and Related Topics (Proc. Univ. of Georgia Inst., 1961), Prentice-Hall, Englewood Cliffs, N. J., 1962, pp. 213-216. MR 25 \#3539.

8. F. HosoKaWA, "On $\nabla$-polynomials of links," Osaka Math. J., v. 10, 1958, pp. 273282. MR 21 \#1606. $37 \# 302$.

9. B. HuPPERT, Endliche Gruppen. I, Springer-Verlag, Berlin and New York, 1967. MR

10. S. Kinoshita \& H. Terasaka, “On unions of knots,” Osaka Math. J., v. 9, 1957, pp. 131-153. MR 20 \#4846.

11. W. Magnus, A. KarRass \& D. Solitar, Combinatorial Group Theory: Presentations of Groups in Terms of Generators and Relations, Pure and Appl. Math., vol. 13, Interscience, New York, 1966. MR 34 \#7617.

12. W. Magnus \& A. Peluso, “On knot groups," Comm. Pure Appl. Math., v. 20, 1967, pp. 749-770. MR 36 \#5930.

13. K. REIDEMEISTER, Knotentheorie, Chelsea, New York, 1948.

14. H. SchUBERT, "Ưber eine numerische Knoten Invariante," Math. Z., v. 61, 1954, pp. 245-288. MR 17, 292. 18, 498 .

15. H. SchUBERT, "Knoten mit zwei Brücken," Math. Z., v. 65, 1956, pp. 133-170. MR 